\title{
Accelerated free-breathing 3D T1 $\rho$ cardiovascular magnetic resonance using multicoil compressed sensing
}

Srikant Kamesh Iyer ${ }^{1}$, Brianna Moon², Eileen Hwuang ${ }^{2}$, Yuchi Han ${ }^{3}$, Michael Solomon², Harold Litt ${ }^{1,3}$ and Walter R. Witschey ${ }^{1 *}$

\begin{abstract}
Background: Endogenous contrast T1 $\rho$ cardiovascular magnetic resonance (CMR) can detect scar or infiltrative fibrosis in patients with ischemic or non-ischemic cardiomyopathy. Existing 2D T1 $\rho$ techniques have limited spatial coverage or require multiple breath-holds. The purpose of this project was to develop an accelerated, freebreathing 3D T1 $\rho$ mapping sequence with whole left ventricle coverage using a multicoil, compressed sensing (CS) reconstruction technique for rapid reconstruction of undersampled k-space data.

Methods: We developed a cardiac- and respiratory-gated, free-breathing 3D T1 $\rho$ sequence and acquired data using a variable-density $k$-space sampling pattern $(A=3)$. The effect of the transient magnetization trajectory, incomplete recovery of magnetization between T1 $\rho$-preparations (heart rate dependence), and k-space sampling pattern on T1 $\rho$ relaxation time error and edge blurring was analyzed using Bloch simulations for normal and chronically infarcted myocardium. Sequence accuracy and repeatability was evaluated using $\mathrm{MnCl}_{2}$ phantoms with different $\mathrm{T} 1 \rho$ relaxation times and compared to $2 \mathrm{D}$ measurements. We further assessed accuracy and repeatability in healthy subjects and compared these results to 2D breath-held measurements.
\end{abstract}

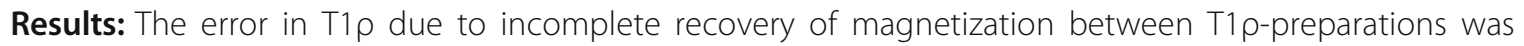
$\mathrm{T} 1 \rho_{\text {healthy }}=6.1 \%$ and $\mathrm{T} 1 \rho_{\text {infarct }}=10.8 \%$ at $60 \mathrm{bpm}$ and $\mathrm{T} 1 \rho_{\text {healthy }}=13.2 \%$ and $\mathrm{T} 1 \rho_{\text {infarct }}=19.6 \%$ at $90 \mathrm{bpm}$. At a heart rate of $60 \mathrm{bpm}$, error from the combined effects of readout-dependent magnetization transients, k-space undersampling and reordering was $\mathrm{T} 1 \rho_{\text {healthy }}=12.6 \%$ and $\mathrm{T} 1 \rho_{\text {infarct }}=5.8 \%$. CS reconstructions had improved edge sharpness (blur metric $=0.15$ ) compared to inverse Fourier transform reconstructions (blur metric $=0.48$ ). There was strong agreement between the mean $\mathrm{T} 1 \rho$ estimated from the $2 \mathrm{D}$ and accelerated $3 \mathrm{D}$ data $\left(R^{2}=0.99 ; P<0.05\right)$ acquired on the $\mathrm{MnCl}_{2}$ phantoms. The mean $\mathrm{R} 1 \rho$ estimated from the accelerated $3 \mathrm{D}$ sequence was highly correlated with $\mathrm{MnCl}_{2}$ concentration $\left(R^{2}=0.99 ; P<0.05\right)$. 3D T1 $\rho$ acquisitions were successful in all human subjects. There was no significant bias between undersampled 3D T1 $\rho$ and breath-held 2D T1 $\rho$ (mean bias $=0.87$ ) and the measurements had good repeatability $\left(\mathrm{COV}_{2 \mathrm{D}}=6.4 \%\right.$ and $\left.\mathrm{COV}_{3 \mathrm{D}}=7.1 \%\right)$.

Conclusions: This is the first report of an accelerated, free-breathing 3D T1 $\rho$ mapping of the left ventricle. This technique may improve non-contrast myocardial tissue characterization in patients with heart disease in a scan time appropriate for patients.

Keywords: Parametric mapping, T1 p, Compressed sensing, Multicoil reconstruction, Fast minimization, Endogenous contrast techniques

\footnotetext{
*Correspondence: witschey@pennmedicine.upenn.edu

'Department of Radiology, Perelman School of Medicine, University of

Pennsylvania, Philadelphia, PA 19104, USA

Full list of author information is available at the end of the article
}

(c) The Author(s). 2018 Open Access This article is distributed under the terms of the Creative Commons Attribution 4.0 International License (http://creativecommons.org/licenses/by/4.0/), which permits unrestricted use, distribution, and reproduction in any medium, provided you give appropriate credit to the original author(s) and the source, provide a link to the Creative Commons license, and indicate if changes were made. The Creative Commons Public Domain Dedication waiver (http://creativecommons.org/publicdomain/zero/1.0/) applies to the data made available in this article, unless otherwise stated. 


\section{Background}

In cardiomyopathy patients, myocardial fibrosis is prognostic for greater risk of sudden cardiac death, development of heart failure, and greater rates of rehospitalization [1]. Patients with suspected fibrosis are often referred for gadolinium enhanced cardiovascular magnetic resonance (CMR) due to the late accumulation and slow release of gadolinium-based contrast agents (GBCAs) from the fibrotic tissue [2,3]. For many patients with advanced renal disease, GBCAs are contraindicated due to poor filtration of the contrast agent and retention of gadolinium in the body [4]. To address this problem, there has been a recent resurgence of endogenous contrast imaging and relaxation time mapping methods, such as $\mathrm{T} 1[5,6]$ and $\mathrm{T} 1 \rho \mathrm{CMR}$, for non-GBCA assessment of myocardial fibrosis [7-9].

Cardiac T1 $\rho$ CMR, also called $\mathrm{T} 1$ in the rotating frame, uses a moderate amplitude radiofrequency pulse (i.e. spin locking pulse), as the contrast-generation mechanism [10, 11]. The spin locking pulse has been shown in preclinical validation experiments and in patients to suppress background sources of transverse relaxation, and improve the dynamic range between water ${ }^{1} \mathrm{H}$ relaxation in fibrotic and normal myocardial tissues [12-15]. However, existing 2D cardiac T1 $\rho$ methods have limited spatial coverage and resolution, limiting the detection of myocardial fibrosis [10, 11]. Multiple long breath-holds are required for whole heart coverage. This can lead to misalignment of images, repeat scans and an increase in patient discomfort. A 3D method was implemented for large animals [11], but is not appropriate for patients due to long scan times and use of ventilator gating in anesthetized animals.

Compressed sensing (CS) $[16,17]$ can be used to reconstruct high quality images from undersampled k-space data by leveraging signal sparsity in a relevant image transform domain. CS uses iterative techniques to minimize the reconstruction cost functional, balancing data fidelity and regularization. Some CS T1 $\rho$ techniques have been developed for applications such as imaging the brain [18], knee [19, 20] and spine [18]. Depending on the application, different sparsifying transforms and reconstruction formulations were developed to reconstruct artifact free images from the undersampled data. Although these techniques have shown high quality reconstruction for their specific applications, their reconstruction speed is relatively slow. These reconstruction formulations do not use rapid minimization techniques such as Split Bregman [17] or Augmented Lagrangian [21]), which have been shown to accelerate the convergence of CS reconstructions. These alternating direction method of multipliers (ADMM)-based techniques use variable substitution to separate the sparsity and data fidelity terms and this decoupling allows for accelerated convergence.
A recent a study [22] compared several sparsity and low rank models and found that spatial-temporal finite difference reconstructions performed well at reconstructing high quality images from undersampled knee cartilage data. To accelerate convergence, approaches based on the application of fast iterative shrinkagethresholding algorithm (FISTA) [23] and its variants were developed. The reconstruction speed of the sparse and low rank methods might be further improved by using Split Bregman or Augmented Lagrangian implementations, though no such methods are currently available for accelerated 3D T1 $\rho$ CMR. Additionally, the performance of these CS techniques has not been prospectively assessed for $3 \mathrm{D}$ cardiac $\mathrm{T} 1 \rho$ mapping or in other organs. There are no methods available for accelerated 3D myocardial T1 $\rho$ mapping.

We aim to develop an accelerated free-breathing whole heart 3D T1 $\rho$ mapping technique using CS. We use a modified $\mathrm{k}$-space ordering technique to reduce cardiac and respiratory motion degradation, and develop a rapid reconstruction formulation based on a novel combination of Split Bregman -based variable substitution and Fourier minimization for multi-coil CS. We analyze the sources of $\mathrm{T} 1 \rho$ error using Bloch simulations and phantoms. Finally, we show feasibility, accuracy and repeatability of the technique for left ventricle T1 $\rho$ mapping.

\section{Methods}

\section{Bloch simulations}

We performed Bloch simulations to measure the accuracy of 3D T1 $\rho$ maps for numerical phantoms with T1 $\rho$ relaxation times that approximated normal $(\mathrm{T} 1 \rho=60$ $\mathrm{ms}$ and $\mathrm{T} 1=1000 \mathrm{~ms})$ and infarcted $(\mathrm{T} 1 \rho=120 \mathrm{~ms}$ and $\mathrm{T} 1=1200 \mathrm{~ms}$ ) myocardium [11, 24], and assessed the effect of variations of the measured k-space signal and data undersampling on image blurring. We implemented a 3D T1p-prepared balanced steady-state free-precession (bSSFP) sequence consisting of a T1 $p$ preparation period, a bSSFP magnetization signal transient stabilization period and bSSFP readout [10]. The T1 $\rho$ preparation consisted of a composite radiofrequency (RF) pulse utilizing a spin echo, spin lock (SL) $\left(90_{\mathrm{x}}-\mathrm{SL}_{\mathrm{y}}-180_{\mathrm{y}}-\mathrm{SL}_{-\mathrm{y}}-90_{-\mathrm{x}}\right)$ [25]. Prior to spatial encoding, a second magnetization preparation was performed using a bSSFP flip angle ramp [10]. Spatial encoding was performed using a bSSFP readout. The simulation parameters were flip angle $=70^{\circ}$, number of segments $/$ readout echoes per heartbeat $\left(\mathrm{N}_{\mathrm{seg}}\right)=48$, number of shots/heartbeats $\left(\mathrm{N}_{\text {shot }}\right)=24$, heart rate $=60 \mathrm{bpm}$, spin lock $\mathrm{B}_{1}=500 \mathrm{~Hz}$, $\mathrm{TE} / \mathrm{TR}=1.4 / 2.8 \mathrm{~ms}$, and the time between $\mathrm{T} 1 \rho$ preparation was $2 \mathrm{~s}$. Bloch simulations were performed by solving the piecewise time-independent matrix solution to the Bloch equations [10]. 
Undersampling pattern and $\mathrm{k}$-space sample ordering

Undersampled $\mathrm{k}$-space data $(\mathrm{A}=3)$ was acquired using a variable density sampling pattern, fully sampled along $k_{x}$, while directions $k_{y}, k_{z}$ were undersampled using a bell shaped polynomial variable density distribution [26] given by

$$
P\left(\mathrm{k}_{y}, k_{z}\right)=\left(1-r\left(k_{y}, k_{z}\right)\right)^{p}
$$

where $r$ is the normalized distance from the k-space center. Samples close to the center had high sampling probability while samples further away had low sampling probability. The polynomial order $(p)$ controlled the kspace sampling density.

A k-space ordering technique was devised to minimize motion inconsistencies between heart beats due to respiratory motion. The total number of sampled k-space locations were divided evenly into the total number of shots $\left(\mathrm{N}_{\text {shot }}\right)$, where each shot had $\mathrm{N}_{\text {seg }}$ sampled locations. Points that were closest to the center of $k_{y}-k_{z}$ were sampled in the earliest shot. Within a shot, a linear (column wise) sorting order was used.

\section{Image blur and T1 $\rho$ accuracy}

We determined the combined effects of the sequence magnetization response function and data undersampling on image quality (blur) and $\mathrm{T} 1 \rho$ relaxation time accuracy using a cylindrical phantom simulation. Image quality was analyzed using the blur metric [27]. The blur metric is normalized to the range $[0,1]$, and a large blur metric corresponds to more blurring of the edges in the image. The relaxation time estimated from the reconstructed images was compared with the known T1 $\rho$ value used for the Bloch simulations and the error was calculated.

\section{Phantom experiments}

Experiments were performed on phantoms to compare the accuracy and repeatability of the accelerated 3D sequence to the 2D T1 $\rho$ sequence [28]. Five cylindrical phantoms were prepared in $15-\mathrm{ml}$ conical tubes of distilled water and 0.01, 0.03, 0.05, 0.07 and $0.09 \mathrm{mM}$ $\mathrm{MnCl}_{2}$ and the outer compartment contained tap water. For both $2 \mathrm{D}$ and $3 \mathrm{D}$ T1 $\rho$ acquisitions, data were acquired using 7 spin lock times (TSLs) $(\mathrm{TSL}=2,10,18$, $26,34,42,50 \mathrm{~ms})$. The sequence was triggered with a simulated heart rate of $60 \mathrm{bpm}$ and data were acquired with $100 \%$ navigator efficiency. This corresponds to a scan time of $0.8 \mathrm{~min} / \mathrm{TSL}$ for the 3D acquisitions and 14 $\mathrm{s}$ for the $2 \mathrm{D}$ acquisition. Other scan parameters for the phantom experiments were the same as detailed below in the human subject sections. Data acquisition was repeated three times to test for repeatability.

\section{Healthy human studies}

The study was approved by the institutional review board (IRB) of the University of Pennsylvania and informed consent was acquired from the subjects prior to data acquisition. Eight prospectively undersampled $(\mathrm{A}=3)$ 3D T1 $\rho$ datasets were acquired from six healthy subjects on a $1.5 \mathrm{~T}$ scanner (Avanto; Siemens Healthineers, Erlangen, Germany) equipped with an 18 channel anterior body and posterior spine RF coil arrays using a T1 $\rho$-prepared bSSFP sequence and a spin echo, SL T1 $\rho$ pulse cluster $\left(90_{x}-\mathrm{SL}_{\mathrm{y}}-180_{\mathrm{y}}-\mathrm{SL}_{-\mathrm{y}}-90_{-\mathrm{x}}\right)$ and $\mathrm{B}_{1}=500 \mathrm{~Hz}$. T1rho weighted images were acquired at 12 TSL's (TSL $=2,5,8,10,15,20,25,30,35,40,45,50 \mathrm{~ms})$. The images were acquired at a $1.9 \times 1.9 \times 6 \mathrm{~mm}^{3}$ spatial resolution using a $(192 \times 144 \times 24)$ acquisition matrix. Other imaging parameters were $\mathrm{TR} / \mathrm{TE}=2.8 / 1.53 \mathrm{~ms}$, $\mathrm{BW}=898 \mathrm{~Hz} /$ pixel and flip angle $=70^{\circ}$, and asymmetric echo. Data were acquired using electrocardiogram (ECG)-gating to end-systole and respiratory navigatorgating to end-expiration. ECG gating and navigator triggering was performed to reduce the effects of cardiac and respiratory motion. The end-systolic phase was determined by examining the cardiac motion from a short-axis cine scan and the beginning of the spatial encoding was adjusted such that $\mathrm{T} 1 \rho$-weighted images were acquired during end-systole, which corresponds to maximum wall thickness. Imaging was performed every other heartbeat. For a $60 \mathrm{bpm}$ heart rate and 50\% navigator efficiency, the scan time for acquiring one T1 $\rho$-weighted 3D dataset was $\sim 1.6 \mathrm{~min}$. The navigator efficiency varied between patients and also between the different TSL weighted scans within a patient. The mean navigator efficiency for acquired datasets was $(54.4 \% \pm 12)$ and the total scan time for the human scans was $\sim 18 \mathrm{~min}$. A $2 \mathrm{D} \mathrm{T} 1 \rho$ scan was performed on a mid-ventricular slice for each human subject. The $2 \mathrm{D}$ T1 $\rho$ images were acquired with a $1.9 \times 1.9 \mathrm{~mm}$ resolution and $8 \mathrm{~mm}$ slice thickness. T1 $\rho$ weighted images were acquired at 7 TSL's (TSL $=2,10,18,26,34,42,50 \mathrm{~ms}$ ). Data acquisition as performed every other heart beat. A total of 14 heart beats were required for the $2 \mathrm{D}$ breath-held acquisitions. The scan for the $2 \mathrm{D}$ acquisition varied between subjects depending on their heart rate. For a nominal heart-rate of $60 \mathrm{bpm}$, the scan time for the $2 \mathrm{D}$ breath-held scan was $14 \mathrm{~s}$. The $3 \mathrm{D}$ acquisition was repeated twice on two subjects to test for repeatability. The pulse sequence for ECG and navigator gated 3D T1 $\rho$ and the accelerated k-space sampling order schema is shown in Fig. 1.

\section{CMR image reconstruction}

Undersampled k-space data was reconstructed using a multicoil CS formulation. The unconstrained problem is given by: 


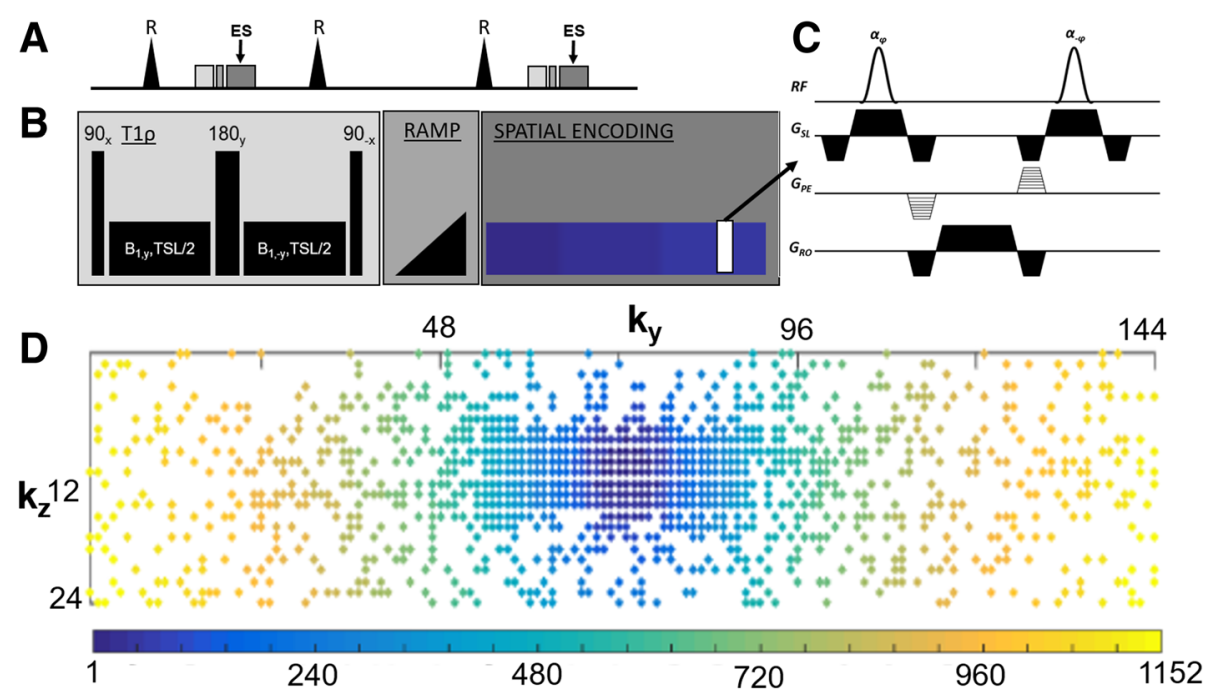

Fig. 1 Pulse sequence for electrocardiogram (ECG) and navigator gated 3D T1 $\rho$ and accelerated k-space sampling order schema. a Image gating is synchronized with end-systole to achieve maximum myocardial wall thickness during this cardiac no motion period. Three heartbeats are shown. Sampling occurs every other heartbeat to allow for longitudinal relaxation of magnetization. $\mathbf{b} T 1 \rho$ and magnetization stabilization (ramp) followed by spatial encoding of a k-space subset is performed during the end-systolic time. c Enhanced view of the balanced steady-state free-precession kernel used during ramp and spatial encoding. $\mathbf{d} \mathrm{K}$-space sample ordering is shown. In this example, a subset of kspace (i.e. $48 \mathrm{k}$-space phase encoding lines) are acquired per heartbeat. K-space sample ordering is performed to assure that center spatial encoding frequencies are collected in the same heartbeat as much as possible. The color bar indicates the k-space ordering index

$$
C=\frac{\mu}{2} \sum_{i=1}^{N c}\left\|E C_{i} m-k_{i}\right\|_{2}^{2}+\lambda\left|\nabla_{x y z} m\right|_{1}
$$

Here $E$ is the 3D encoding matrix, that includes the sampling pattern and the Fourier operator, $C$ is the coil sensitivity map, $N_{c}$ the total number of coils, $k$ is the measured k-space data and $\nabla_{x y z}$ is the 3D spatial gradient operator used to apply the 3D total variation (TV) constraint [17]. The cost functional is rapidly minimized using the application of the Split Bregman based variable substitution technique [17] by applying the following two variable substitutions, $S=\nabla_{x y z} m$ and $P_{i}=C_{i} m$. Enforcing the two variable substitutions using Split Bregman, eq. (2) is rewritten as

$$
\begin{array}{r}
\min P_{i}, m, S, T, Q_{i} \frac{\mu}{2} \sum_{i=1}^{N c} \begin{array}{l}
\left\|E P_{i}-k_{i}\right\|_{2}^{2}+\frac{\alpha}{2} \sum_{i=1}^{N c} \\
\left\|P_{i}-C_{i} m-Q_{i}\right\|_{2}^{2}+\lambda
\end{array} \\
|S|_{1}+\frac{\beta}{2}\left\|S-\nabla_{x y z} m-T\right\|_{2}^{2}
\end{array}
$$

Here $S$ and $P_{i}$ are the surrogate variables and $Q_{i}$ and $T$ come from optimizing the Bregman distance [17]. The variables $P_{i}$ and $m$ are present only in $\mathrm{L}_{2}$ norm terms and are decoupled from the $\mathrm{L}_{1}$ norm term.

$$
\min P_{i} \frac{\mu}{2} \sum_{i=1}^{N c}\left\|E P_{i}-k_{i}\right\|_{2}^{2}+\frac{\alpha}{2} \sum_{i=1}^{N c}\left\|P_{i}-C_{i} m-Q_{i}\right\|_{2}^{2}
$$

$$
\min m \frac{\beta}{2}\left\|S-\nabla_{x y z} m-T\right\|_{2}^{2}+\frac{\alpha}{2} \sum_{i=1}^{N c}\left\|P_{i}-C_{i} m-Q_{i}\right\|_{2}^{2}
$$

The terms $Q_{i}$ and $T$ are minimized using a simple linear update step [17]. The iterations were terminated when a convergence criterion was met or the maximum set of iterations was reached. Pseudocode of the reconstruction algorithm is provided as Additional file 1: Figure S1.

The reconstruction method was implemented in MATLAB $^{\text {TM }}$ (Mathworks, Natick Massachusetts, USA) and tested on a computer with an intel Xeon E5-2603 $\mathrm{CPU}$ with a processor frequency of $1.8 \mathrm{GHz}, 8$ cores and a total memory of 64GB. The weights for the cost functional were chosen based on a test dataset. The weights were chosen as $\mu=0.6, \lambda=0.9, \alpha=0.1$ and $\beta=0.1$. The set of weights chosen allowed for the rapid convergence of the cost functional. The maximum number of iterations was set to 100 and the iteration was terminated when a set tolerance level was reached. 


\section{CMR parametric mapping}

Motion correction using diffeomorphic registration [29] was performed prior to quantification. This has been shown to improve the quality of $\mathrm{T} 1 \rho$ fit [10]. T1 $\rho$ maps were generated from the images by fitting a two parameter signal model [30] given by

$$
S=S_{0} e^{\left(\frac{-T S L}{T 1 \rho}\right)}
$$

where $\mathrm{S}_{0}$ is the initial magnetization and TSL is the spin-lock pulse duration.

\section{Statistical analysis}

All descriptive quantities are reported as mean and standard deviation (mean $\pm \mathrm{SD}$ ). Comparisons of $2 \mathrm{D}$ and 3D T1 $\rho$ phantom scans were performed using linear regression, intra-class correlation coefficient (ICC), coefficient of variation $(\mathrm{COV})$ and Bland-Altman plots. The mean $\mathrm{R} 1 \rho$ for the five phantoms was compared with the concentration of $\mathrm{MnCl}_{2}$ using linear regression and Pearson's $r$. For the in vivo cardiac acquisitions, myocardial T1 $\rho$ maps were divided into six regions and the mean, SD and COV were computed.

\section{Results}

\section{Bloch simulations and k-space ordering}

The error in T1 $\rho$ due to incomplete recovery of magnetization between $\mathrm{T} 1 \rho$ preparations was $6.1 \%$ (normal) and $10.8 \%$ (infarct) at $60 \mathrm{bpm}$ and $13.2 \%$ (normal) and19.6\% (infarct) at $90 \mathrm{bpm}$. The dependence of T1 $\rho$ relaxation error on heart rate is shown in Fig. 2a for normal and infarcted tissue. A delay of $3 \mathrm{~s}$ between T1 $\rho$-preparations reduced the percentage error in normal tissue to less than $2 \%$ of its true value and less than $4.2 \%$ in infarct.

The Bloch-simulated magnetization response is shown in Fig. 2b. The fully sampled image is shown in Fig. 2c and an example of an undersampling pattern modulated by the readout-dependent magnetization transients is shown in Fig. 2d. The images reconstructed from the undersampled and magnetization transience modulated k-space data are shown in Fig. 2e. The image reconstructed from the undersampled data using the inverse Fourier transform (IFT) suffers from edge blurring (blur metric $=0.48$ ) while the image reconstructed using 3D TV constraints has a sharper edge profile (blur metric $=0.15$ ). This result is further visualized in Fig. $2 \mathrm{e}$ using the plot of a vertical line across the image, the
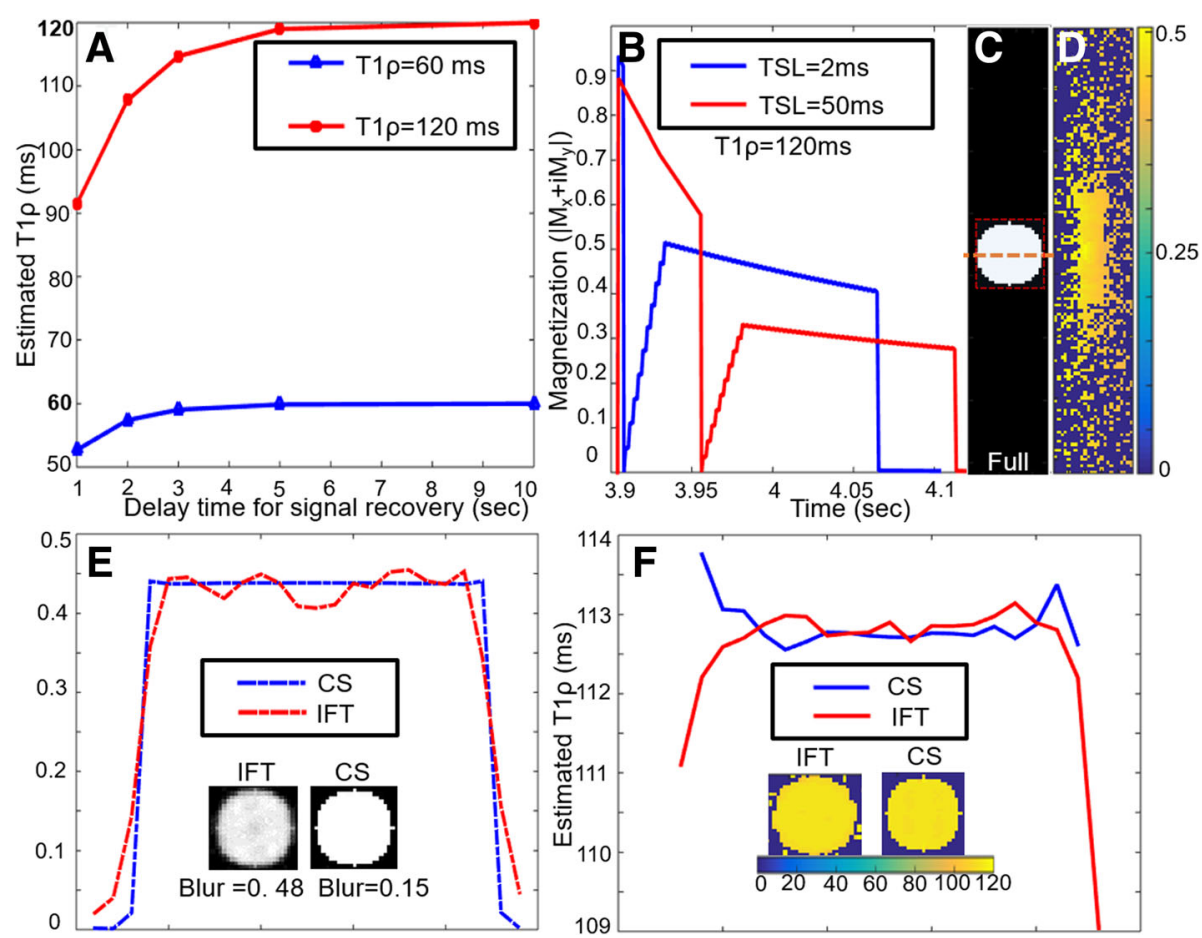

Fig. 2 Results from Bloch simulation of the accelerated 3D T1 $\rho$ acquisition. a Curve showing the dependence of T1 $\rho$ relaxation error on heart rate. $\mathbf{b}$ The magnetization response curves showing the transverse magnetizations for TSL $=2 \mathrm{~ms}$ and $50 \mathrm{~ms}, \mathbf{c}$ the fully sampled input image, $\mathbf{d}$ the k-space undersampling pattern modulated by the transient magnetization, e The plot of intensities across a horizontal line, (the location is shown using an orange dotted line in Fig. 2c) for the images reconstructed from the undersampled data using inverse Fourier transform (IFT) and using 3D TV constraints. The CS reconstruction has sharper edges as compared to IFT reconstruction. $\mathbf{f}$ The plot of estimated T1 $\rho$ values across the horizontal line for the T1 $\rho$ map estimated from the IFT and 3D CS reconstruction 
location is shown using an orange dotted line in Fig. 2c. The T1 $\rho$ map estimated from the IFT and CS reconstruction are shown in Fig. 2f. As seen in the plot of a line across the estimated T1 $\rho$ maps in Fig. 2f, blurring of edges in IFT reconstructions lead an increased error in the estimated $\mathrm{T} 1 \rho$ values at the edges of the cylinder, akin to the partial volume effect. The mean T1 $\rho$ estimated from IFT was $(112.9 \pm 0.2) \mathrm{ms}$ and from CS reconstruction was $(113.1 \pm 0.2) \mathrm{ms}$. For the simulation of the normal myocardium, the mean $\mathrm{T} 1 \rho$ estimated from IFT was $(67.4 \pm 0.1) \mathrm{ms}$ and from CS reconstruction was $(67.1 \pm 0.1) \mathrm{ms}$. The combined error from incomplete magnetization recovery, readout-dependent magnetization transients and undersampled k-space acquisition was $12 \%$ for healthy myocardium and 5.8\% for infarct at $60 \mathrm{bpm}$.

\section{Phantom experiments}

To test the accuracy and repeatability of the accelerated $3 \mathrm{D} \mathrm{T} 1 \rho$ scan, we prepared phantoms with different $\mathrm{T} 1 \rho$ relaxation times, scanned them three times, and reconstructed images and analyzed estimated T1 $\rho$ maps and compared to $2 \mathrm{D}$ T1 $\rho$ (Fig. 3a-d). There was excellent agreement between 2D and 3D T1 $\rho$ acquisitions $\left(R^{2}=0.99, P<0.05\right.$; ICC $\left.=0.99\right)$; Fig. $\left.3 e\right)$ and we were unable to detect a significant bias (Fig. 3f). R1p was highly correlated with $\mathrm{MnCl}_{2}$ concentration $\left(R^{2}=0.99 ; \mathrm{P}<0.05\right.$; Fig. $\left.3 \mathrm{~g}\right)$. The mean T1 $\rho$ from the three repeat acquisitions are reported in Additional file 2: Table S1. We did not detect a significant difference between the mean T1 $\rho$ estimated from the 3 repeat acquisitions $(\mathrm{ICC}=0.99)$.

\section{Human experiments}

Example T1 $\rho$ images reconstructed using the multicoil 3D total variation formulation is shown in Fig. 4a-d. The convergence criterion was achieved in approximately 50 $\mathrm{s}$, as shown in Additional file 3: Figure S2. A comparison of $\mathrm{T} 1 \rho$ weighted images reconstructed from the proposed undersampled 3D acquisition and a breath-held 2D acquisition is shown in Additional file 4: Figure S3. High quality T1 $\rho$ weighted images were reconstructed using the proposed multicoil $3 \mathrm{D} \mathrm{TV}$ reconstruction formulation.
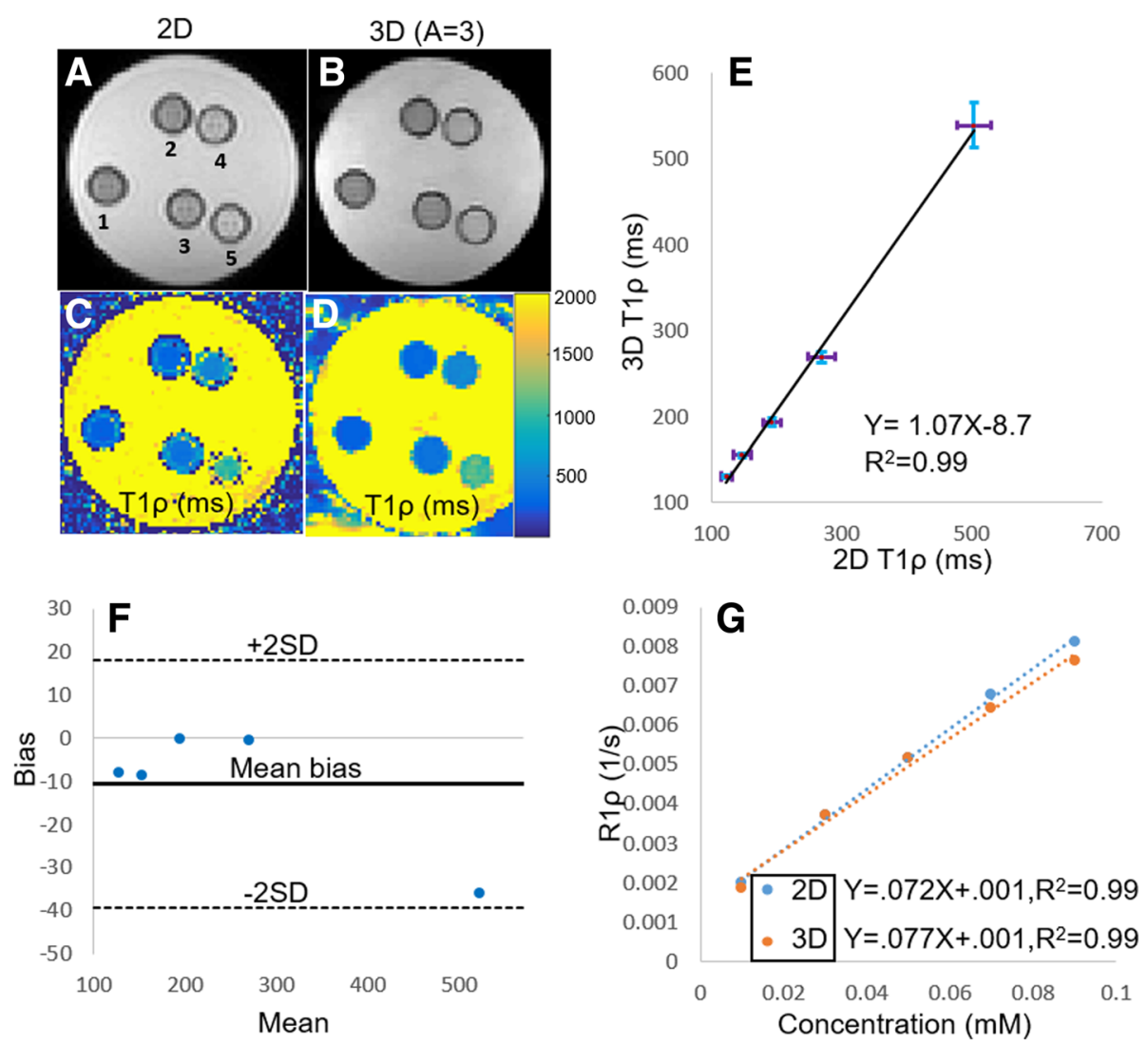

Fig. $3 \mathrm{~T} 1 \mathrm{p}$-weighted images (TSL = $50 \mathrm{~ms}$ ) (a, b) and maps (c, d) of saline phantoms doped with $1-5 \mathrm{wt} \% \mathrm{MnCl}_{2}$. Images obtained using 2D single-shot $(\mathbf{a}, \mathbf{c})$ and accelerated $(\mathrm{A}=3)$ compressed sensing $(\mathbf{b}, \mathbf{d})$ acquisitions. e Plot comparing the correlation between mean T1 $\rho$ estimated from the $2 \mathrm{D}$ and accelerated $3 \mathrm{D}$ images for the five $\mathrm{MnCl}_{2}$ doped phantoms. $\mathbf{f}$ Bland-Altman plot comparing the mean $\mathrm{T} 1 \rho$ (in $\mathrm{ms}$ ) estimated from the $2 \mathrm{D}$ and accelerated $3 \mathrm{D}$ images. $\mathbf{g}$ Plot comparing correlation between $\mathrm{R} 1 \rho$ and concentration of $\mathrm{MnCl}_{2}$ 

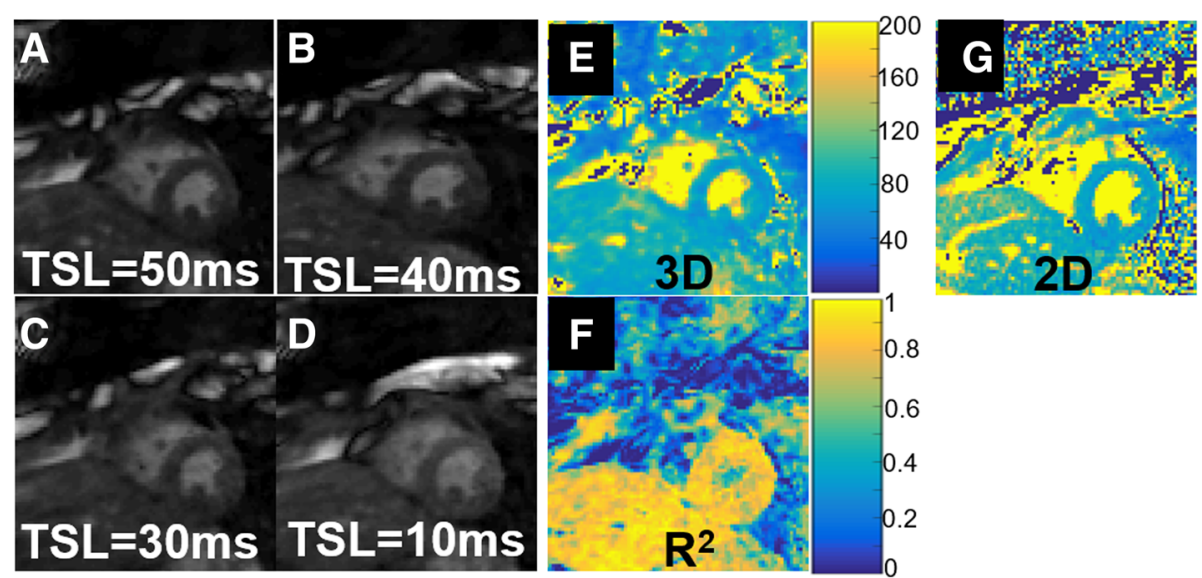

Fig. 4 Results from an in-vivo human acquisition. T1 $\rho$ weighted images reconstructed using the proposed multicoil 3D total variation formulation is shown in (a-d). The myocardial T1 $\rho$ map (e) showed good uniformity and low least squares fit error (f). T1 $\rho$ map with the breath-held 2D sequence acquired at the same slice position is shown in $(\mathbf{g})$. The mean T1 $\rho$ estimated from the free-breathing 3D scans and breath-held 2D were $67.9 \pm 4.5 \mathrm{~ms}$ and $71.4 \pm 6.5 \mathrm{~ms}$, respectively

As expected in healthy subjects, the myocardial T1 $\rho$ map (Fig. 4e) showed good uniformity. A comparison with breath-held 2D T1 $\rho$ map, acquired at the same slice position, is shown in Fig. 4g. The T1 $\rho$ maps estimated from the free-breathing 3D scans and breath-held 2D scans showed good correspondence (3D T1 $\rho=(67.9 \pm$ $4.5 \mathrm{~ms})$ and $2 \mathrm{D} \mathrm{T} 1 \rho=(71.4 \pm 6.5 \mathrm{~ms}))$. The mean $\mathrm{T} 1 \rho$, $\mathrm{COV}$ and average navigator efficiency for the healthy subjects is reported in Additonal file 5: Table S2. The mean myocardial T1 $\rho$ estimated from the $2 \mathrm{D}$ and $3 \mathrm{D}$ scans matched well and there was no significant bias (mean bias $=0.87$ ). The Bland-Altman plot based comparison of the estimated mean $\mathrm{T} 1 \rho$ is shown in Additional file 6: Figure S4. High quality images were reconstructed from the two repeat scans on healthy subjects. The comparison of the mean myocardial T1p showed that the proposed 3D technique is repeatable and the coefficient of variation of the myocardial T1 $\rho$ for $3 \mathrm{D}$ and $2 \mathrm{D}$ acquisitions on average were 7.1 and $6.4 \%$ respectively.

\section{Discussion}

In this study, we developed an accelerated, free-breathing 3D T1 $\rho$ mapping technique that can provide full coverage of the left ventricle in human subjects. The significance of this work compared to existing CS $3 \mathrm{D}$ T $1 \rho$ mapping techniques $[18-20,22,31]$ is that we have developed a T1 $\rho$ pulse sequence with variable-density undersampling on a clinical CMR scanner and prospectively assessed its accuracy and repeatability for cardiac imaging in phantoms and human subjects. Additionally, the reconstruction uses a novel combination of Split Bregman variable substitution and Fourier minimization for rapid reconstruction in a clinical setting.
A major contribution of this work is the analysis of the 3D T1 $\rho$ sequence using Bloch simulations to study the effect of incomplete magnetization recovery (heart rate), data undersampling and transient signal decay on edge blurring and relaxation time accuracy. Existing CS T1 $\rho$ techniques have been analyzed only in the setting of fully sampled k-space data that has been retrospectively undersampled and could not account for these factors. Since the k-space signal is dependent on magnetization transients, motion, and k-space ordering, it is necessary to prospectively acquire undersampled data to assess feasibility in human subjects. Existing methods assumed a uniform magnetization response and did not account for the transient effects of spatial encoding and k-space ordering on measured relaxation times.

The k-space ordering pattern used in this study tries to mitigate cardiac motion by acquiring k-space locations closest to the center of k-space in the same heartbeat/shot. Within a shot, a linear (column wise) sorting order was used to reduce the amount of eddy-current artifact due to rapid switching of gradients. In preliminary analysis, we found that by not splitting the low-frequency components of the k-space into multiple parts that were acquired over several heart beats, the motion artifacts could be better mitigated (data not shown). Since image quality can be affected by factors like eddy currents and motion, some novel k-space ordering techniques have been suggested [32-34]. In some studies [32, 33], the sampled $\mathrm{k}$-space positions were first sorted based on the angular (radial) location of the points. The points within a shot were then sorted based on the radial distance from the center of $\mathrm{k}$-space. This choice of k-space ordering was performed to minimize eddy current artifacts in applications such as contrast- 
enhanced whole-heart coronary CMR and accelerated 3D cine phase contrast imaging. In [34], the ill-effects of respiratory motion were sought to be mitigated by dynamically changing the location of acquired phase encode based on the location of the navigator.

In this study, a 3D TV constraint was chosen as the sparsifying transform for the CS implementation. This choice of constraint was to utilize the correlations in the data in the three spatial dimensions. The high performance of TV based constraints on undersampled cardiac data has been observed for several applications such as dynamic contrast enhanced cardiac perfusion imaging [35-38], cine imaging [39-41], and late gadolinium enhanced imaging of the left atrium $[26,42]$.

An acceleration factor of $\mathrm{A}=3$ was achieved by using a variable density sampling pattern in this study. Achieving high acceleration factors for free-breathing 3D mapping of the heart is a challenge due to the presence of cardiac and respiratory motion. In addition, Bloch simulation showed that variation in heart rate also has an effect on the estimated T1p.

Rapid multicoil CS minimization techniques have been proposed using methods such as FISTA [23], variable splitting [43], proximal operators [44], fast composite splitting algorithm (FCSA) [45, 46], SB/AL [17, 47], or a combination of techniques for applications such as cine imaging, dynamic contrast enhanced perfusion imaging and imaging of the brain. A detailed list of these techniques and their applications are shown in Additional file 7: Table S3. Depending on the application, these techniques have been developed to handle problems that arise due to a specific application of the type of constraint and nature of the data encoding matrix. The rapid reconstruction formulation developed here does not use FISTA-based iterative re-weighting for minimizing the quadratic $\mathrm{L}_{2}$ norm terms $[37,43,44]$. The Split Bregman- based variable substitution developed here uses one fewer variable substitution compared to [47] and does not need complex matrix factorization techniques used in $[47,48]$. A detailed comparison of some of the existing rapid multicoil CS techniques is provided in [37].

CS 3D T1 $\rho$ techniques $[18-20,22,31]$ have been developed for applications such as imaging of the knee cartilage, spine and brain. Since these techniques have been developed for different applications, each with their own set of challenges, there are differences in the sequence parameters such as acquired resolution, field-of-view, slice thickness, number of TSLs, and spatial encoding; in turn, these parameters affect the reconstruction performance. The parameter choices are customized to the needs of the application. A summary of these methods is discussed in Additional file 8: Table S4. In [18], a combination of principal component analysis and dictionary learning was used to reconstruct undersampled $\mathrm{k}$-space data. The technique, called PANDA-T1 $\rho$, was tested on retrospectively undersampled human brain and spine data with acceleration factors up to $A=4$. A limitation of PANDA-T1 $\rho$ is the long reconstruction time and high computational complexity due to the need to train the dictionary at each iteration. In another study [19], a reconstruction technique developed for knee imaging used a sequential combination of a data-driven parallel imaging and the use of 2D spatial TV sparsifying transform. The CS reconstructions were performed on each coil data separately and minimized using a non-linear conjugate gradient solver. This technique was tested on two retrospectively undersampled in vivo human knee datasets for a net acceleration factor of 2.2, although slightly higher acceleration factors were reported on ex vivo porcine knee. The accelerated T1 $\rho$ mapping technique developed for imaging cartilage in [20] used an algorithm that alternated between local support detection in the space of principal components and joint image reconstruction and sensitivity estimation in SENSE (JSENSE). Feasibility of the technique was shown for acceleration factors up to 3.5 retrospectively in human knee cartilage. Accelerated simultaneous T2 and T1 $\rho$ MRI has been achieved using a blind compressed sensing (BCS) technique for brain mapping [31]. A limitation of this technique is the increased computational complexity and reconstruction time. A comparison of several CS reconstruction techniques with sparse and low rank models for T1 $\rho$ MRI has been recently reported [22]. The techniques were implemented using different variants of FISTA and combined with fast gradient projection (FGP) based algorithms. These were tested on phantom data and retrospectively undersampled in vivo knee cartilage data. The results showed that the implementations with spatiotemporal finite difference as sparsity constraint performed best for high acceleration factors.

Respiratory motion and cardiac motion are unique challenges in CMR that remain major factors in determining what acceleration factors may be achieved. Compared to applications such as imaging knee cartilage, human brain and spine, CMR parametric mapping has challenges in achieving very high acceleration rates. For example, for applications such as accelerated free-breathing 3D T1 mapping of the heart [49-51] the reported acceleration rates are comparable to what we have achieved. Existing accelerated 3D T1 $\rho$ techniques for non-CMR applications are analyzed by retrospectively undersampling fully sampled data. This approach is not suitable for in vivo cardiac applications as the fully sampled data is susceptible to intershot motion. Based on these factors, we chose to acquire prospectively undersampled data with an acceleration factor $(\mathrm{A}=3)$, for which free-breathing 3D T1 $\rho$ mapping of the heart was feasible. 
In order to estimate the feasibility of achieving higher acceleration factor for $3 \mathrm{D}$ cardiac $\mathrm{T} 1 \rho$ mapping, we performed experiments by retrospectively undersampling $\mathrm{A}=3$ data to $A=4$ using a binary mask and compared the reconstructed images to $A=3$. The results (in Additional file 9: Figure S5) show that in principle, it is feasible to achieve $A=4$, though with a marginal loss in image quality. Compared to $\mathrm{A}=3$ images, $\mathrm{A}=4 \mathrm{im}$ ages show additional smoothing of edges and loss of features. This shows that it will be difficult to achieve higher acceleration factors in clinical applications.

\section{Limitations}

The study could be further improved by increasing the number of datasets. Unlike retrospectively undersampled techniques that can perform analysis at different acceleration factors $(\mathrm{A}=2,2.5$ and 3$)$, we did not acquire data at multiple acceleration factors due to the limited availability of patient scan time. Since the technique performs well at $\mathrm{A}=3$, the proposed technique should also work at lower acceleration factors. The effect of higher acceleration factors on free breathing 3D T1 $\rho$ would have to be further analyzed.

One major criterion that affected image quality was the constant change of the navigator position due to change in the patients breathing pattern or patient movement inside the scanner. One way to mitigate this problem would be to change the navigator acceptance window and position adaptively for each TSL acquired, though this could cause an increase in the total scan time.

Another limitation of the study was the long scan time needed to acquire the data. To estimate good quality T1 $\rho$ maps, images acquired using 12 TSL's were used during curve fitting. This corresponded to a scan time of $\sim 18 \mathrm{~min}$ at a $50 \%$ navigator efficiency. The scan time could be further reduced by increasing the navigator acceptance window width, though this could come at a cost of decrease in image quality of the reconstructed images due to motion.

\section{Future work}

The reconstruction time of the technique can be accelerated by running on GPU's or implementing on C ++ instead of MATLAB. In addition, the reconstruction time could be further reduced by using coil compression techniques $[52,53]$ to reduce the total number of coils used for the reconstructions. The reconstructed image quality can be further improved by using additional constraints along the parameter dimension. The sampling pattern used to acquire the data from different TSL's would have to be randomized so that artifacts do not overlap in the temporal dimension and sparsity can be effectively imposed. This can be applied in conjunction with motion compensation techniques to mitigate for cardiac motion between different TSL's. This may also allow for further reduction in scan time by increasing the acceleration factor. One major drawback of simultaneously using constraints in the parameter dimension and spatial dimensions would be the increase in the reconstruction time, which would impede in the application of the reconstruction pipeline in a clinical framework. Though the technique has been developed for 3D T1 $\rho$ cardiac mapping, it is possible to extend the ideas from this work to other parametric mapping technique application with minimal modifications.

\section{Conclusions}

This study shows that the proposed combination of multicoil 3D TV reconstruction and variable density based k-space undersampling allows for free-breathing T1 $\rho$ mapping of the whole heart. High quality T1 $\rho$ weighted images were rapidly reconstructed using the proposed SB based technique. The mean T1 $\rho$ estimated using the proposed technique matched well with the currently used 2D T1 $\rho$ mapping technique and there was no significant bias. This technique has the potential for clinical application of free-breathing T1 $\rho$ mapping.

\section{Additional files}

Additional file 1: Figure S1. Pseudocode of the reconstruction algorithm. (DOCX 37 kb)

Additional file 2: Table S1. Results comparing the repeatability of T1 $\rho$ phantoms experiment. Mean, standard deviation (SD), coefficient of variation (COV) and results from the intra-class correlation coefficient (ICC) test is reported. (DOCX $12 \mathrm{~kb}$ )

Additional file 3: Figure S2. Plot showing the convergence of the cost functional. The relative change between successive iterations drops below $0.01 \%$ at the 48 th iteration in $\sim 51 \mathrm{~s}$. (DOCX $735 \mathrm{~kb}$ )

Additional file 4: Figure S3. Figure comparing T1 $\rho$ weighted images from 2D ((A)-(F)) and 3D acquisitions ((G)-(L)). Good quality images were reconstructed from the undersampled $3 \mathrm{D}$ acquisitions. (DOCX $78 \mathrm{~kb}$ )

Additional file 5: Table S2. Results from in-vivo human datasets comparing 2D and 3D scans. Mean T1 $\rho$ (ms), COV and average navigator efficiency are reported. (DOCX $15 \mathrm{~kb}$ )

Additional file 6: Figure S4. Bland-Altman plot comparing the mean $\mathrm{T} 1 \rho$ (in ms) estimated from 2D and accelerated 3D images. (DOCX $42 \mathrm{~kb}$ )

Additional file 7: Table S3. An overview of some of the existing techniques that have been developed to rapidly minimize cost functionals of the form $C=\frac{\mu}{2} \sum_{i=1}^{N c}\left\|E C_{i} m-k_{i}\right\|^{2}+\lambda\left|\varphi_{1} m\right|_{1}+\eta\left|\varphi_{2} m\right|_{1}$ is provided. (DOCX $54 \mathrm{~kb}$ )

Additional file 8: Table S4. An overview of the existing techniques proposed for accelerated CS T1 $\rho$ reconstructions. (DOCX $18 \mathrm{~kb}$ )

Additional file 9: Figure S5. Comparison of reconstructions from prospectively undersampled $A=3$ data and $A=4$ data that was created by retrospectively undersampling the $A=3$ data using a binary mask (shown in (E)). The images reconstructed from $A=4$ data ((B) and (D) have blurred edges, as compared to images reconstructed from $A=3$ data (in (A) and (C)). (DOCX $202 \mathrm{~kb}$ ) 


\section{Abbreviations}

ADMM: Alternating direction method of multipliers; BCS: Blind compressed sensing; bSSFP: Balanced steady-state free precession; CMR: Cardiovascular magnetic resonance; COV: Coefficient of variation; CS: Compressed sensing; ECG: Electrocardiogram; FCSA: Fast composite splitting algorithm; FGP: Fast gradient projection; FISTA: Fast iterative shrinkage-thresholding algorithm; GBCA: Gadolinium based contrast agent; ICC: Intra-class correlation coefficient; IFT: Inverse fourier transform; JSENSE: Joint image reconstruction and sensitivity estimation in sensitivity encoding; PANDA: Principle component analysis and dictionary learning; RF: Radiofrequency; SD: Standard deviation; SL: Spin lock; TSL: Spin lock time; TV: Total variation

\section{Acknowledgements}

Not applicable.

\section{Funding}

The authors acknowledge support for research through UL1TR0018787, R00HL108157, Institute for translational medicine and therapeutics (ITMAT) transdisciplinary program in translational medicine and therapeutics, McCabe Foundation, and W.W. Smith Foundation.

\section{Availability of data and materials}

The datasets are available from the corresponding author on reasonable request.

\section{Authors' contributions}

SKI Study design, analysis and interpretation of data, statistics, drafting of the manuscript. BM Study design, analysis and interpretation of data, revising the manuscript, EH Analysis and interpretation of data statistics, revising the manuscript. YH Study design, Interpretation of data, revising the manuscript. MS revising the manuscript, data analysis and interpretation. HL Study design, analysis and interpretation of data, revising the manuscript. WRTW study design, analysis and interpretation of data, statistics, drafting of the manuscript. All authors read and approved the final manuscript.

\section{Ethics approval and consent to participate}

All datasets were acquired under the approval of the Institutional Review Board of the University of Pennsylvania.

\section{Consent for publication}

All datasets were acquired with informed consent.

\section{Competing interests}

The authors declare that they have no competing interests.

\section{Publisher's Note}

Springer Nature remains neutral with regard to jurisdictional claims in published maps and institutional affiliations. Dr. Matthias Stuber served as a JCMR Guest Editor for this manuscript.

\section{Author details}

${ }^{1}$ Department of Radiology, Perelman School of Medicine, University of Pennsylvania, Philadelphia, PA 19104, USA. ${ }^{2}$ Department of Bioengineering, University of Pennsylvania, Philadelphia, PA 19104, USA. ${ }^{3}$ Department of Medicine, University of Pennsylvania, Philadelphia, PA 19104, USA.

Received: 4 May 2018 Accepted: 13 November 2018 Published online: 10 January 2019

\section{References}

1. Kuruvilla S, Adenaw N, Katwal AB, Lipinski MJ, Kramer CM, Salerno M. Late gadolinium enhancement on cardiac magnetic resonance predicts adverse cardiovascular outcomes in nonischemic cardiomyopathy: a systematic review and meta-analysis. Circ Cardiovasc Imaging. 2014;7:250-8.

2. Kim RJ, Chen E-L, Lima JAC, Judd RM. Myocardial Gd-DTPA kinetics determine MRI contrast enhancement and reflect the extent and severity of myocardial injury after acute Reperfused infarction. Circulation. 1996;94:3318.

3. Abdel-Aty H, Zagrosek A, Schulz-Menger J, Taylor AJ, Messroghli D, Kumar A, Gross M, Dietz R, Friedrich MG. Delayed enhancement and T2-weighted cardiovascular magnetic resonance imaging differentiate acute from chronic myocardial infarction. Circulation. 2004;109:2411.
4. Marckmann P, Skov L, Rossen K, Dupont A, Damholt MB, Heaf JG, Thomsen HS. Nephrogenic systemic fibrosis: suspected causative role of Gadodiamide used for contrast-enhanced magnetic resonance imaging. J Am Soc Nephrol. 2006;17:2359-62.

5. Messroghli DR, Greiser A, Fröhlich M, Dietz R, Schulz-Menger J. Optimization and validation of a fully-integrated pulse sequence for modified look-locker inversion-recovery (MOLLI) T1 mapping of the heart. J Magn Reson Imaging. 2007;26:1081-6.

6. Messroghli DR, Radjenovic A, Kozerke S, Higgins DM, Sivananthan MU, Ridgway JP. Modified look-locker inversion recovery (MOLLI) for highresolution T1 mapping of the heart. Magn Reson Med. 2004;52:141-6.

7. Witschey WRT, Borthakur A, Elliott MA, Fenty M, Sochor MA, Wang C, Reddy R. T1 $\rho$-prepared balanced gradient echo for rapid 3D T1 $\rho$ MRI. J Magn Reson Imaging. 2008;28:744-54.

8. Redfield AG. Nuclear magnetic resonance saturation and rotary saturation in solids. Phys Rev. 1955;98:1787-809.

9. Wang L, Yuan J, Zhang SJ, Gao M, Wang YC, Wang YX, Ju S. Myocardial T1rho mapping of patients with end-stage renal disease and its comparison with T1 mapping and T2 mapping: a feasibility and reproducibility study. J Magn Reson Imaging. 2016;44:723-31.

10. Berisha S, Han J, Shahid M, Han Y, Witschey WRT. Measurement of myocardial T1 $\rho$ with a motion corrected, parametric mapping sequence in humans. PLoS One. 2016;11:e0151144.

11. Witschey WRT, Zsido GA, Koomalsingh K, Kondo N, Minakawa M, Shuto T, McGarvey JR, Levack MM, Contijoch F, Pilla JJ, et al. In vivo chronic myocardial infarction characterization by spin locked cardiovascular magnetic resonance. J Cardiovasc Magn Reson. 2012;14:37.

12. Han Y, Liimatainen T, Gorman RC, Witschey WR. Assessing myocardial disease using T1rho MRI. Curr Cardiovasc Imaging Rep. 2014;7:9248.

13. Musthafa HS, Dragneva G, Lottonen L, Merentie M, Petrov L, Heikura T, YlaHerttuala E, Yla-Herttuala S, Grohn O, Liimatainen T. Longitudinal rotating frame relaxation time measurements in infarcted mouse myocardium in vivo. Magn Reson Med. 2013;69:1389-95.

14. van Oorschot JW, El Aidi H, Jansen of Lorkeers SJ, Gho JM, Froeling M, Visser F, Chamuleau SA, Doevendans PA, Luïten PR, Leiner T, Zwanenburg JJ. Endogenous assessment of chronic myocardial infarction with T(1 rho)-mapping in patients. J Cardiovasc Magn Reson. 2014;16:104. https://doi.org/10.1186/s12968-014-0104-y.

15. van Oorschot JW, Visser F, Eikendal AL, Vonken EP, Luijten PR, Chamuleau SA, Leiner T, Zwanenburg JJ. Single breath-hold T1 rho-mapping of the heart for endogenous assessment of myocardial fibrosis. Investig Radiol. 2016. https://doi.org/10.1097/RLI.0000000000000261.

16. Lustig M, Donoho D, Pauly JM, Sparse MRI. The application of compressed sensing for rapid MR imaging. Magn Reson Med. 2007;58:1182-95.

17. Goldstein T, Osher S. The Split Bregman method for L1-regularized problems. SIAM J Imaging Sci. 2009;2:323-43.

18. Zhu Y, Zhang Q, Liu Q, Wang YXJ, Liu X, Zheng H, Liang D, Yuan J. PANDAT1 p: integrating principal component analysis and dictionary learning for fast T1 $\rho$ mapping. Magn Reson Med. 2015;73:263-72.

19. Pandit P, Rivoire J, King K, Li X. Accelerated T1 $\rho$ acquisition for knee cartilage quantification using compressed sensing and data-driven parallel imaging: a feasibility study. Magn Reson Med. 2016;75:1256-61.

20. Zhou Y, Pandit P, Pedoia V, Rivoire J, Wang Y, Liang D, Li X, Ying L. Accelerating t1 $\rho$ cartilage imaging using compressed sensing with iterative locally adapted support detection and JSENSE. Magn Reson Med. 2016;75:1617-29.

21. Tai X-C, Wu C. Augmented Lagrangian method, dual methods and Split Bregman iteration for ROF model. In: Proceedings of the Second International Conference on Scale Space and Variational Methods in Computer Vision. Voss, Norway: Springer-Verlag; 2009. p. 502-13.

22. Zibetti MWW, Sharafi A, Otazo R, Regatte RR. Accelerating 3D-T1 $\rho$ mapping of cartilage using compressed sensing with different sparse and low rank models. Magn Reson Med. 2018;0:1-18.

23. Beck A, Teboulle M. A Fast Iterative Shrinkage-thresholding algorithm for linear inverse problems. SIAM J Imaging Sci. 2009;2:183-202.

24. Witschey WRT, Pilla JJ, Ferrari G, Koomalsingh K, Haris M, Hinmon R, Zsido G, Gorman JH, III, Gorman RC, Reddy R. Rotating Frame Spin Lattice Relaxation in a Swine Model of Chronic, Left Ventricular Myocardial Infarction. Magn Reson Med. 2010;64(5):1453-60.

25. Witschey WR 2nd, Borthakur A, Elliott MA, Mellon E, Niyogi S, Wallman DJ, Wang C, Reddy R. Artifacts in T1 rho-weighted imaging: compensation for $\mathrm{B}(1)$ and $\mathrm{B}(0)$ field imperfections. J Magn Reson. 2007; 186:75-85. 
26. Kamesh lyer S, Tasdizen T, Burgon N, Kholmovski E, Marrouche N, Adluru G, DiBella E. Compressed sensing for rapid late gadolinium enhanced imaging of the left atrium: a preliminary study. Magn Reson Imaging. 34:846-54.

27. Crete F, Dolmiere T, Ladret $P$, Nicolas M. The blur effect: perception and estimation with a new no-reference perceptual blur metric. Proc. SPIE 6492, Human Vision and Electronic Imaging XII, 64920l (12 February 2007); https:// doi.org/10.1117/12.702790.

28. Berisha S, Han J, Shahid M, Han Y, Witschey WR. Measurement of myocardial T1rho with a motion corrected, Parametric Mapping Sequence in Humans. PLoS One. 2016;11:e0151144.

29. Avants BB, Tustison NJ, Song G, Cook PA, Klein A, Gee JC. A reproducible evaluation of ANTs similarity metric performance in brain image registration. Neurolmage. 2011;54:2033-44.

30. Feng L, Srichai MB, Lim RP, Harrison A, King W, Adluru G, Dibella EVR, Sodickson DK, Otazo R, Kim D. Highly-accelerated real-time cardiac cine MRI using k-t SPARSE-SENSE. Magn Reson Med. 2013;70:64-74.

31. Bhave S, Lingala SG, Johnson CP, Magnotta VA, Jacob M. Accelerated whole-brain multi-parameter mapping using blind compressed sensing. Magn Reson Med. 2016;75:1175-86.

32. Basha TA, Akçakaya M, Goddu B, Berg S, Nezafat R. Accelerated threedimensional cine phase contrast imaging using randomly undersampled echo planar imaging with compressed sensing reconstruction. NMR Biomed. 2015:28:30-9.

33. Akçakaya M, Basha TA, Chan RH, Rayatzadeh H, Kissinger KV, Goddu B, Goepfert LA, Manning WJ, Nezafat R. Accelerated contrast-enhanced wholeheart coronary MRI using low-dimensional-structure self-learning and thresholding. Magn Reson Med. 2012;67:1434-43.

34. Jhooti P, Wiesmann F, Taylor AM, Gatehouse PD, Yang GZ, Keegan J, Pennell DJ, Firmin DN. Hybrid ordered phase encoding (HOPE): an improved approach for respiratory artifact reduction. J Magn Reson Imaging. 1998;8:968-80.

35. Adluru G, Awate SP, Tasdizen T, Whitaker RT, DiBella EVR. Temporally constrained reconstruction of dynamic cardiac perfusion MRI. Magn Reson Med. 2007;57:1027-36

36. Adluru G, Whitaker RT, Dibella EVR. Spatio-temporal constrained reconstruction of sparse dynamic contrast enhanced radial MRI data. In: 2007 4th IEEE international symposium on biomedical imaging: from Nano to macro; 2007. p. 109-12.

37. Iyer SK, Tasdizen T, Likhite D, DiBella E. Split Bregman multicoil accelerated reconstruction technique: a new framework for rapid reconstruction of cardiac perfusion MRI. Med Phys. 2016;43:1969-81.

38. Chen C, Li Y, Axel L, Huang J. Real time dynamic MRI with dynamic Total variation. In: Cham: Springer International Publishing; 2014. p. 138-45.

39. Usman M, Ruijsink B, Nazir MS, Cruz G, Prieto C. Free breathing whole-heart 3D CINE MRI with self-gated Cartesian trajectory. Magn Reson Imaging. 2017;38:129-37.

40. Montesinos P, Abascal JFPJ, Cussó L, Vaquero JJ, Desco M. Application of the compressed sensing technique to self-gated cardiac cine sequences in small animals. Magn Reson Med. 2014;72:369-80.

41. Feng L, Axel L, Chandarana H, Block KT, Sodickson DK, Otazo R. XD-GRASP: Golden-angle radial MRI with reconstruction of extra motion-state dimensions using compressed sensing. Magn Reson Med. 2016;75:775-88.

42. Adluru G, Chen L, Kim S-E, Burgon N, Kholmovski E, Marrouche N, DiBella EVR. 3D late gadolinium enhancement imaging of the left atrium with stack of stars and compressed sensing. J Magn Reson Imaging. 2011;34:1465-71.

43. Ye $X$, Chen $Y$, Lin W, Huang F. Fast MR image reconstruction for partially parallel imaging with arbitrary k-space trajectories. IEEE Trans Med Imaging. 2011;30:575-85.

44. Montefusco LB, Lazzaro D, Papi S, Guerrini C. A Fast Compressed Sensing approach to 3D MR image reconstruction. IEEE Trans Med Imaging. 2011;30:1064-75.

45. Huang J, Zhang S, Metaxas D. Efficient MR image reconstruction for compressed MR imaging. Med Image Anal. 2011;15:670-9.

46. Jiang M, Jin J, Liu F, Yu Y, Xia L, Wang Y, Crozier S. Sparsity-constrained SENSE reconstruction: an efficient implementation using a fast composite splitting algorithm. Magn Reson Imaging. 2013;31:1218-27.

47. Ramani S, Fessler JA. Parallel MR image reconstruction using augmented Lagrangian methods. IEEE Trans Med Imaging. 2011;30:694-706.

48. Bilen C, Wang Y, Selesnick IW. High-speed compressed sensing reconstruction in dynamic parallel MRI using augmented Lagrangian and parallel processing. IEEE J Emerg SelectTop Circ Syst. 2012;2:370-9.
49. Coniglio A, Di Renzi P, Vilches Freixas G, Della Longa G, Santarelli A, Capparella R, Nardiello B, Loiudice C, Bianchi S, D'Arienzo M, Begnozzi L. Multiple 3D inversion recovery imaging for volume T1 mapping of the heart. Magn Reson Med. 2013;69:163-70.

50. Nordio G, Henningsson M, Chiribiri A, Villa ADM, Schneider T, Botnar RM. 3D myocardial T1 mapping using saturation recovery. J Magn Reson Imaging. 2017:46:218-27.

51. Weingärtner $S$, Akçakaya M, Roujol S, Basha T, Stehning C, Kissinger KV, Goddu B, Berg S, Manning WJ, Nezafat R. Free-breathing post-contrast three-dimensional T1 mapping: volumetric assessment of myocardial T1 values. Magn Reson Med. 2015;73:214-22.

52. Adluru G, DiBella E. Compression(2): compressed sensing with compressed coil arrays. J Cardiovasc Magn Reson. 2012;14:P242.

53. Buehrer M, Pruessmann KP, Boesiger $P$, Kozerke S. Array compression for MRI with large coil arrays. Magn Reson Med. 2007:57:1131-9.

\section{Ready to submit your research? Choose BMC and benefit from:}

- fast, convenient online submission

- thorough peer review by experienced researchers in your field

- rapid publication on acceptance

- support for research data, including large and complex data types

- gold Open Access which fosters wider collaboration and increased citations

- maximum visibility for your research: over $100 \mathrm{M}$ website views per year

At BMC, research is always in progress.

Learn more biomedcentral.com/submissions 